\title{
A Fuzzy Integrated Approach for Evaluating Third-Party Logistics
}

\author{
Amir abbas Shojaie, Amir Reza Soltani, and Mohammad Reza Soltani
}

\begin{abstract}
Third-party logistics (3PL) selection is one of the most important decisions for companies to outsource some part of their activities and concentrate more on its own core competencies. Because of the significant role of 3PL in total performance of the supply chain, its selection is a critical act. This is the most meaningful motivation to generate new solutions to select the best 3PL using appropriate criteria. These criteria and their weights are often full of ambiguities. This makes fuzzy logic a more suitable approach to solve this problem. In this paper, we aim to present a new evaluation method using a combined MCDM approach. Fuzzy weighted and credibility theories are utilized to determine the weights of criteria, and the graph theory and $\mathrm{ABC}$ inventory classification are used to priorities alternatives. To demonstrate applicability of the proposed approach, a real case 3PL selection problem is provided.
\end{abstract}

Index Terms - Third-party, fuzzy set theory, logistics, graph theory.

\section{INTRODUCTION}

A third-party logistics provider (3PL) is a firm that provides service to its customers of outsourced logistics services for part, or all of their supply chain management functions. This facts enhance accomplishing logistic functions in the markets. This outsourcing approach is an important decision that will make many benefits for the main company. It will allow to the outsourcing company to focus on its core competencies and increase its internal efficiency [1]. Fig. 1 represents the process of 3PL, schemtically.

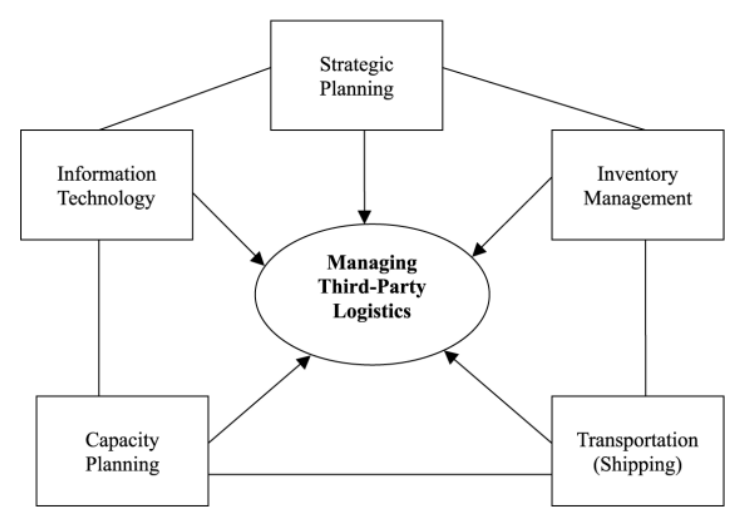

Fig. 1. Scheme of third-party logistics process.

Consequently, an appropriate selected provider would really have a positive impact on the total performance of the company. [2] Such providers are known as third-party logistics (3PL), playing the role of a bridge between the main company and its environment. They may do various functions such as transportation, warehousing, inventory

Manuscript received November 1, 2015; revised May 1, 2016.

Amir Abbas is with School of Industrial Engineering, Islamic Azad University, South Tehran Branch, Tehran, Iran (e-mail: amir@ ashojaie.com) management, and information systems. There is plenty of research studying 3PL from different viewpoint, especially about its selection [3], [4].

In this article, we follow to propose a new approach to evaluate 3PL using Graph theory, Fuzzy logic, Credibility theorem and $\mathrm{ABC}$ analysis. At first, the literature is reviewed and then the new method is proposed. Furthermore the proposed method is examined using a real case study. We also applied Perron Frobenius theoremdeveloped firstly by Perron [5] for a matrix with positive entries and then extended by Frobenius [6] for a non-negative case. The theorem measures eigenvectors and eigenvalues of a non-negative entry matrix. It has numerous applications in communication, Markov chains, pricing, etc. On the other hand, calculating eigenvalues of a large matrix plays an important role in several domains, for example fluid dynamics, engineering, physics, chemistry and economics [7]. In the actual world, matrixes are large and complex, and this leads to some numerical problems and errors during calculating their eigenvalues. Among various tools for measuring eigenvalues, the Perron Frobenius theorem[5,6] does not produce these errors. It is useful in producing some eigenvalue features of non-negative entries' matrixes.

The rest of paper is as follows: Next section provides some required definitions and concepts. Section III presnets proposed integrated approach. Section IV provides some numerical illustrations. At end, conclusions and future resaerhes are given.

\section{PRELIMINARIES AND DEFINITIONS}

\section{A. Directed Graph}

A directed graph is used to represent the structure of the multi-cretria decision making (MCDM) problem. The attributes are nodes of the graph, and the links between them are directed arcs. If the direction of an arc is from node $A$ to node $B$, then node $A$ dominates $B$.Let $\mathrm{M}=(\mathrm{V}, \mathrm{E})$ be a graph consisting of a set of vertices $V$ and a set of edges $E$ In this respect, two following definitation of graphs is requred to be defined:

- Path count in directed graph:

There is a directed graph on $n$ nodes with an adjacency matrix $\mathrm{M} \in \mathrm{R}^{\mathrm{n} * \mathrm{n}}$

$$
M_{i j}=\left\{\begin{array}{c}
1, \text { there is an edge from node } j \text { to node } i \\
0, \quad \text { otherwise }
\end{array}\right.
$$

A possible way to evaluate alternatives against a criterion is to find a directed Hamilton route. With respect to the position of alternatives in this route, they should be prioritized. For instance, a directed Hamilton route such as $(3,1,2,4,5,6)$ shows number 3 is the best alternative while number 6 has the least priority. This approach has some difficulties, because in general, there are several Hamilton routes. 
- Defining diameter of a directed graph:

In a strongly connected graph $(\mathrm{M})$, the shortest directed route between $\mathrm{U}$ and $\mathrm{V}$ is the distance between them. It is called $\vec{d}_{M}(U, V)$. Supposing $\mathrm{M}$ as a strongly connected graph with $V \geq 5$ and $A$ as its adjacency matrix, in $\mathrm{A}^{\mathrm{d}+3}$ (with positive entities) $d$ is the directed diameter of M.The result of solving this theorem is;Adjacency matrix $\mathrm{M}$, is initial if and only if $V \geq 4$ and $\mathrm{M}$ be a strongly connected graph.The ith score vectors in tournament $\mathrm{M}$ is:

$$
S_{i}=A^{i} J
$$

Wherein $\mathrm{A}$ is adjacency matrix $\mathrm{M}$ and $\mathrm{J}$ is a column vector with entities equal to one. If matrix $\mathrm{A}$ is initial then based on Perron Frobenius theorem [5], [6] the absolute highest eigenvalue will be a positive number such as $r$ and therefore we have [8];

$$
\lim _{i \rightarrow \infty}\left(\frac{A}{r}\right)^{i} J=S
$$

\section{B. ABC Analysis}

There are many methods for inventory management. One the most popular methods is the ABC inventory classification. The main concept behind it is the Pareto principle and therefore it classifies items into three groups (A, B, and C) based on annual dollar usage of each item. [9] Items in class $A$ are relatively few in numbers but large in annual usage value. While items in class $\mathrm{C}$, are high in numbers but low in annual value. There is another class between these two classes, which constitute class B. In this simple method, control and monitoring policy for class A should be tight [10].Although this classification is easy in use and the most widely employed technique in organizations, it has some limitations. Many companies have various items in their inventory list, but $\mathrm{ABC}$ makes just three kinds of classes for all of them. On the other hand hundreds of items used by companies are not homogenous, while the $\mathrm{ABC}$ classification is only successful when inventory is classified homogenously. [11]

\section{Credibility Theory}

Fuzzy numbers are introduced to express linguistic variables appropriately. In the following, some basic definitions of the fuzzy sets theory will be reviewed briefly from Kaufmann and Gupta [12], Raj and Kumar [13] and Cheng and Lin [3].The fuzzy set theory introduced by Zadeh [14] is suitable for dealing with uncertainty and imprecision associated with information concerning various parameters. Some notions of fuzzy sets and fuzzy numbers are reviewed [2], [15].

In a universal set of discourse $X$, a fuzzy sub set $\mathrm{A}$ of $X$ is defined by a membership function $f_{A}(X)$, wherein $f_{A}(X) \forall x \in X$, indicates the degree of $X$ in A. The degree to which an element belongs to a set is defined by a value between 0 and 1 . If $X$ completely belongs to A, $f_{A}(X)=1$ and if completely not $f_{A}(X)=0$ the higher $f_{A}(X)$ means the greater grade of membership of $X$ in A.

Suppose $\xi$ is a fuzzy variable with membership function $\mu$. Then for any set B of R , the credibility of $\xi \in B$ is defined in(Lin and Liu, 2008) as:

$$
C r(\{\xi \in B\})=\frac{1}{2}\left(\left\{\sup \mu(x)_{x \in B}+1\right\}-\left\{\sup \mu(x)_{x \in B^{c}}\right\}\right)
$$

For any set $\mathrm{B}$, the possibility measure of $\xi \in \mathrm{B}$ was defined by Zadeh [14], that is,

$$
\operatorname{Pos}(\{\xi \in B\})=\sup \mu(x)_{x \in B}
$$

And the necessity measure to be defined[16]:

$$
\operatorname{Nec}(\{x \in B\})=1-\sup \mu(x)_{x \in B^{c}}
$$

A triangular fuzzy variable $\xi$ is one with the following membership function

$$
\mu(x)=\left\{\begin{array}{cc}
\frac{X-a}{b-a} & a \leq X \leq b \\
\frac{X-c}{b-c} & b \leq X \leq c \\
0 & \text { otherwise }
\end{array}\right.
$$

The defining feature of validity, credibility $(\xi \geq X)$ and credibility $(\xi \leq X)$ is as follows:

$$
\begin{gathered}
\operatorname{cr}(\{\xi \geq X\})=\left\{\begin{array}{cc}
1 & X \leq a \\
\frac{a-2 b+X}{2(a-b)} & a \leq X \leq b \\
\frac{X-c}{2(b-c)} & b \leq X \leq c \\
0 & c \leq X
\end{array}\right. \\
C r(\{\xi \leq X\})=\left\{\begin{array}{cc}
0 & X \leq a \\
\frac{X-a}{2(b-a)} & a \leq X \leq b \\
\frac{X+c-2 b}{2(c-b)} & b \leq X \leq c \\
1 & c \leq X
\end{array}\right.
\end{gathered}
$$

Let $\xi_{\mathrm{i}}$ be fuzzy variables with membership functions, $\mu \mathrm{i}$, and let ui be real numbers, $\mathrm{i}=1,2, \ldots, \mathrm{n}$ respectively.Suppose that $\mathrm{f}: \mathrm{R}^{\mathrm{n}} \rightarrow \mathrm{R}$ is a function. Then the credibility of the fuzzy event is characterized by $\mathrm{f}(\xi 1, \xi 2, \ldots, \xi \mathrm{n}) \geq 0$ is: (Liu, 2004)

$$
\begin{gathered}
\operatorname{Cr}\left\{f\left(\xi_{1}, \xi_{2}, \ldots, \xi_{n}\right) \geq 0\right\}= \\
0.5\left(\sup _{u_{1}, \ldots u_{n} \in \mathbb{R}}\left\{\min _{\substack{1 \leq i \leq n \\
\xi_{i}\left(u_{i}\right)}} \mid f\left(u_{1}, \ldots u_{n}\right) \geq 0\right\}+1-\sup _{u_{1}, \ldots u_{n} \in \mathbb{R}}\left\{\min \begin{array}{l}
\mu_{\xi_{i}}\left(u_{i}\right) \\
1 \leq i \leq n
\end{array} \mid f\left(u_{1}, \ldots u_{n}\right) \leq 0\right\}\right)
\end{gathered}
$$

Let $\xi$ be a fuzzy variable. Then its expected value is defined as:

$$
E[\xi]=\int_{0}^{\infty} \operatorname{Cr}(\{\xi \geq X\}) d r-\int_{-\infty}^{0} \operatorname{Cr}(\{\xi \leq X\}) d r
$$

Expected value is one of the most important concepts for fuzzy variables, which gives the center of its distribution [17]. For example according to the definition Eq. (1), the expected value of the triangular fuzzy variable $\xi=(a 1, a 2, a 3)$ is $E[\xi]=(\mathrm{a} 1+2 \mathrm{a} 2+\mathrm{a} 3) / 4$ and for trapezoidal fuzzy variable $\xi=(\mathrm{a} 1, \mathrm{a} 2, \mathrm{a} 3, \mathrm{a} 4)$ is $E[\xi]=(\mathrm{a} 1+\mathrm{a} 2+$ $\mathrm{a} 3+\mathrm{a} 4) / 4 .[13]$

\section{THE PROPOSED APPROACH}

Our model is based on Perron Frobenius [5], [6] and the ABC Inventory classification and includes four steps: 
Step 1: Introduction of Criteria and Attributes

To evaluate 3PLs we need some criteria and attributes based on the context and special situation of each company with $n$ cretria $\left(C_{1}, C_{2}, \ldots, C_{n}\right)$ and $m$ attributes $\left(A_{1}, A_{2}, \ldots, A_{m}\right)$.

Step 2: Calculation of the weights for each criterion

In this step, the fuzzy weight of each criterion is calculated based on Buckley[14]as follow:

$$
\begin{aligned}
& \tilde{r}_{i}=\left[C_{i 1}, C_{i 2}, C_{i 3}, C_{i 4}\right] \quad \forall i=1,2, \ldots, n \\
& \tilde{W}_{i}=\frac{\tilde{r}_{i}}{\tilde{r}_{1} \oplus \cdots \oplus \tilde{r}_{n}}
\end{aligned}
$$

where $r_{i}$ is a trapezoidal fuzzy number and $\tilde{W}_{i}$ is the weight of ith criteria. Then based on the formula (10) the amount of credibility for each criterion is calculated;

$$
\mathrm{E}[\xi]=\frac{\mathrm{a} 1+\mathrm{a} 2+\mathrm{a} 3+\mathrm{a} 4}{4}
$$

If credibility of some criteria is equal in this step, then evaluation will be done by adjusting weight $\left(\tilde{\mathrm{w}}_{\mathrm{i}}^{\prime}\right)$. Indeed, weights resulting from subjective judgment of managers and weight approximation with fuzzy weight finding will be combined and modified. In other words, formula (11) is used: [8]

$$
\widetilde{\mathrm{W}}_{\mathrm{i}}^{\prime}=\frac{\widetilde{W}_{i} \cdot \lambda_{i}}{\sum_{i=1}^{n} \lambda_{i} \cdot \tilde{W}_{i}}, \forall i^{\prime}=1,2, \ldots, n
$$

To calculate final weight of each criterion $\left(\tilde{\mathrm{w}}_{\mathrm{i}}^{\prime}\right)$, we use a combining approach of Buckley's formula $\left(\tilde{W}_{i}\right)$ and the subjective judgment of managers $(\lambda)$ [18]. Using these final weights $\left(\tilde{w}_{i}^{\prime}\right)$ with respect to the amount of credibility, $E[\xi]$, there is a possibility of weights equality. Therefore, we select $\mathrm{C}_{\mathrm{n}}$ criteria based on the amount of credibility.

Step 3: Calculating the matrix of eigenvalues In this step, we calculate the highest eigenvalues $\left(\mathrm{S}_{1}, \mathrm{~S}_{2}, \ldots, \mathrm{S}_{\mathrm{m}}\right)$ based on the Perron- Frobenius theorem[5,6] as below:

$$
\lim _{i \rightarrow \infty}\left(\frac{A_{N}}{r_{N}}\right)^{i} J=S_{N} \quad ; N=1, \ldots, n
$$

Similarly, adjacency matrixes $\left(M_{1}, M_{2}, \ldots, M_{n}\right)$ are calculated based on graphs in step 1. In adjacency matrixes $\left(M_{1}, M_{2}, \ldots, M_{n}\right)$, priority of each alternative against criteria is evaluated considering directions between nodes. $S_{N}$ is a positive eigenvector correspondent to $r_{N}$ of $A_{N}$. Normalized vector, $\overline{S_{N}}$, with summation of all entities equal to one, could be considered as relative priority of alternatives against criteria in adjacency matrices.

Step 4: Calculating final priority of alternatives

After calculating positive vectors, $S_{1}, S_{2}, \ldots, S_{m}$, priority of alternatives is calculated by performing an $\mathrm{ABC}$ analysis several times. In this step, final priority is obtained by changing the percentage of $\mathrm{A}$ and $\mathrm{B}$ the high, moderate important items in class A and B.

\section{The Results}

In this section, we provide a numerical illustration based on the literature to demonstrate the applicability of the proposed methodology. The example is explained according the steps of proposed approach described in previous section.

Step 1: Supposing $m=6$ and $n=4$, in the 3PL evaluation, 26 criteria are selected from Lin and Wang [2] as Table I.

TABLE I: EVALUATION CRITERIA FOR PROVIDER SELECTION

\begin{tabular}{cl}
\hline Symbol & \\
\hline C1 & Price \\
C2 & Financial consideration \\
C3 & Experience in the similar industry \\
C4 & Location \\
C5 & Asset ownership \\
C6 & International scope \\
C7 & Growth forecasts \\
C8 & Market share \\
C9 & Logistics equipment \\
C10 & Optimization capabilities \\
C11 & Logistics information system \\
C12 & EDI capacity \\
C13 & Customer service \\
C14 & On-time shipments and deliveries \\
C15 & Capabilities to handle specific business requirement \\
C16 & Responsiveness \\
C17 & Service quality \\
C18 & Continuous improvement \\
C19 & Value added service \\
C20 & KPI (key performance indicators) measurement and reporting \\
C21 & Accessibility of contact person in urgency \\
C22 & Cultural fit \\
C23 & General repetition \\
C24 & Service cancellation \\
C25 & Human resource policies \\
C26 & Availability of qualified talent \\
\hline &
\end{tabular}

Step 2: Calculating the weight of criteria

Table II is produced based on the procedure explained in step 2.

\begin{tabular}{cl} 
TABLE II: COMPUTING THE WEIGHTS FOR EACH CRITERION \\
\hline Criteria & Aggregated fuzzy weights \\
\hline C1 & $(0.64,0.74,0.8,0.84)$ \\
C2 & $(0.36,0.46,0.48,0.58)$ \\
C3 & $(0.64,0.74,0.74,0.84)$ \\
C4 & $(0.72,0.82,0.84,0.92)$ \\
C5 & $(0.24,0.34,036,0.46)$ \\
C6 & $(0.22,0.32,0.32,0.42)$ \\
C7 & $(0.3,0.4,0.42,0.52)$ \\
C8 & $(0.58,0.68,0.68,0.78)$ \\
C9 & $(0.62,0.72,0.76,0.84)$ \\
C10 & $(0.3,0.4,0.42,0.5)$ \\
C11 & $(0.78,0.88,0.96,0.98)$ \\
C12 & $(0.6,0.7,0.72,0.8)$ \\
C13 & $(0.76,0.86,0.92,0.96)$ \\
C14 & $(0.78,0.88,0.96,0.98)$ \\
C15 & $(0.76,0.86,0.92,0.96)$ \\
C16 & $(0.76,0.86,0.92,0.96)$ \\
C17 & $(0.76,0.86,0.92,0.96)$ \\
C18 & $(0.62,0.72,0.76,0.84)$ \\
C19 & $(0.625,0.725,0.725,0.825)$ \\
C20 & $(0.3,0.4,0.42,0.52)$ \\
C21 & $(0.76,0.86,0.92,0.96)$ \\
C22 & $(0.58,0.68,0.68,0.78)$ \\
C23 & $(0.725,0.825,0.85,0.925)$ \\
C24 & $(0.48,0.58,0.6,0.7)$ \\
C25 & $(0.38,0.48,0.52,0.62)$ \\
C26 & $(0.54,0.64,0.66,0.74)$ \\
\hline &
\end{tabular}

Considering Table II, the amount of credibility is calculated and shown in Table III. 


\begin{tabular}{ccccccc}
\multicolumn{6}{c}{ TABLE III: THE COMPTATIONAL RESULTS OF CREDIBILITY } \\
\cline { 2 - 7 } Criteria & A1 & A2 & A3 & A4 & CR1 & Rank \\
\hline C1 & 0.64 & 0.74 & 0.8 & 0.84 & 0.755 & \\
C2 & 0.36 & 0.46 & 0.48 & 0.58 & 0.47 & \\
C3 & 0.64 & 0.74 & 0.74 & 0.84 & 0.74 & \\
C4 & 0.72 & 0.82 & 0.84 & 0.92 & 0.825 & \\
C5 & 0.24 & 0.34 & 0.36 & 0.46 & 0.35 & \\
C6 & 0.22 & 0.32 & 0.32 & 0.42 & 0.32 & \\
C7 & 0.3 & 0.4 & 0.42 & 0.52 & 0.41 & \\
C8 & 0.58 & 0.68 & 0.68 & 0.78 & 0.68 & \\
C9 & 0.62 & 0.72 & 0.76 & 0.84 & 0.735 & \\
C10 & 0.3 & 0.4 & 0.42 & 0.5 & 0.405 & \\
C11 & 0.78 & 0.88 & 0.96 & 0.98 & 0.9 & 1 \\
C12 & 0.6 & 0.7 & 0.72 & 0.8 & 0.705 & \\
C13 & 0.76 & 0.86 & 0.92 & 0.96 & 0.875 & 2 \\
C14 & 0.78 & 0.88 & 0.96 & 0.98 & 0.9 & 1 \\
C15 & 0.76 & 0.86 & 0.92 & 0.96 & 0.875 & 2 \\
C16 & 0.76 & 0.86 & 0.92 & 0.96 & 0.875 & 2 \\
C17 & 0.76 & 0.86 & 0.92 & 0.96 & 0.875 & 2 \\
C18 & 0.62 & 0.72 & 0.76 & 0.84 & 0.735 & \\
C19 & 0.625 & 0.725 & 0.725 & 0.825 & 0.725 & \\
C20 & 0.3 & 0.4 & 0.42 & 0.52 & 0.41 & \\
C21 & 0.76 & 0.86 & 0.92 & 0.96 & 0.875 & 2 \\
C22 & 0.58 & 0.68 & 0.68 & 0.78 & 0.68 & \\
C23 & 0.725 & 0.825 & 0.85 & 0.925 & 0.83125 & \\
C24 & 0.48 & 0.58 & 0.6 & 0.7 & 0.59 & \\
C25 & 0.38 & 0.48 & 0.52 & 0.62 & 0.5 & \\
C26 & 0.54 & 0.64 & 0.66 & 0.74 & 0.645 &
\end{tabular}

Because of the equality of ranks and the amount of credibility in Table III for some criteria (C11, C13, C14, C15, $\mathrm{C} 16, \mathrm{C} 17$ and $\mathrm{C} 21$ ), we calculated another rank for them. Because we want to use four high important criteria $(n=4)$ they were selected based on the amount of credibility using the Eq. (10). Between all criteria, four criteria, C17, C13, C14 and $\mathrm{C} 17$, are in the top of the list with the highest amount of credibility. It should be noted that $\lambda$ is a subjective judgment of experts.

Step 3: Adjacency graphs for alternatives considering C17, C13, C14 and C17 are calculated. E.g. we represent adjacency graph $\mathrm{C} 14$ for alternative $\mathrm{A} 2$ in Fig. 2.

Adjacency matrixes $M_{1}$ are obtained from Fig 2 as below:

$$
M_{1}=\left[\begin{array}{llllll}
0 & 1 & 0 & 1 & 1 & 1 \\
0 & 0 & 0 & 1 & 1 & 1 \\
1 & 1 & 0 & 1 & 0 & 0 \\
0 & 0 & 0 & 0 & 1 & 1 \\
0 & 0 & 1 & 0 & 0 & 1 \\
0 & 0 & 1 & 0 & 0 & 0
\end{array}\right]
$$

In a similar manner other adjacency matrices M2, M3 and M4 and eigenvalues (S1-S4) are calculated based on the graphs. In M1, A1 dominates over A2, A4, A5 and A6 from the viewpoint of $\mathrm{C}_{14}$, but is beaten by $\mathrm{A} 3$. For instance, in C14 there is $\mathrm{r}=2.232$ and $\overline{S_{14}}=(0.238,0.164,0.231,0.113,0.150,0.104)$. Therefore, based on this method of evaluation, priorities of alternatives against $\mathrm{C} 14$ are 1, 3, 2, 5, 4, and 6 . Similarly positive vectors of S2-S4 for other criteria are calculated and shown in Table

\section{6.}

Step 4: In this step, after calculating positive vectors $\left(\mathrm{S}_{1}, \mathrm{~S}_{2}, \mathrm{~S}_{3}, \mathrm{~S}_{4}\right)$, we rank alternatives using the $\mathrm{ABC}$ analysis as shown in Table 6 .

As a result, after four iterations of the $\mathrm{ABC}$ analysis and changing the percentage of $\mathrm{A}$ and $\mathrm{B}$ classes, the priorities are finalized as below: $\mathrm{A} 4>\mathrm{A} 2>\mathrm{A} 3>\mathrm{A} 5>\mathrm{A} 1>\mathrm{A} 6$. In this step, alternatives are grouped and prioritized in classes (A, B and $\mathrm{C})$, and the best alternative is put in class A. Therefore, in the provider evaluation problem in the 3PL selection, the alternative A4 is selected.

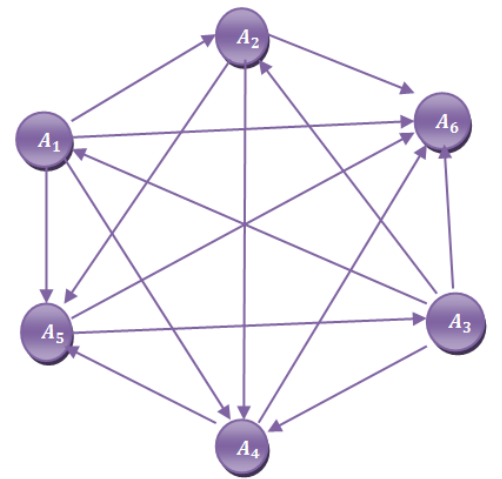

Fig. 2. Adjacency graph C14 for alternative A1, A2... A6.

\begin{tabular}{|c|c|c|c|c|c|c|c|c|}
\hline $\begin{array}{c}\text { Attri } \\
.\end{array}$ & $\mathbf{s}_{1}$ & $\mathbf{S}_{2}$ & $\mathbf{S}_{3}$ & $\mathbf{S}_{4}$ & $\begin{array}{c}\% \\
\text { unit }\end{array}$ & $\begin{array}{c}\% \\
\text { Value }\end{array}$ & $\begin{array}{l}\text { Cumul } \\
\text { ative } \\
\text { Value }\end{array}$ & 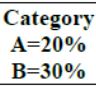 \\
\hline A1 & 0.23 & 0.21 & 0.35 & 0.5 & 6.23 & 21.61 & 21.61 & C \\
\hline A2 & 0.16 & 0.53 & 0.51 & 0.75 & 22.1 & 17.96 & 39.57 & B \\
\hline A3 & 0.23 & 0.75 & 0.42 & 0.41 & 14.9 & 22.71 & 62.28 & B \\
\hline A4 & 0.11 & 0.43 & 0.61 & 0.12 & 30.3 & 27.19 & 89.47 & A \\
\hline A5 & 0.15 & 0.61 & 0.63 & 0.35 & 15.0 & 6.70 & 96.17 & $\mathrm{C}$ \\
\hline A6 & 0.10 & 0.36 & 0.35 & 0.38 & 11.5 & 3.83 & 100 & $\mathrm{C}$ \\
\hline Total & & & & & \multicolumn{4}{|c|}{$\mathrm{A} 4>\mathrm{A} 2=\mathrm{A} 3>\mathrm{A} 1=\mathrm{A} 5=\mathrm{A} 6$} \\
\hline
\end{tabular}

\section{CONCLUSION}

Due to the rapid growth of industries and increased global competition, firms must take care of all processes of business. Outsourcing is a solution to help the main company to concentrate on its own core competencies. It improves the total performance of the supply chain if the provider selects appropriately. Therefore, the selection of the 3PL should be done carefully. In this problem, the decision-maker should select the 3PL using different criteria subjectively. It should be noted that the weights of these criteria are not well known and it will increase the vagueness of the problem. Therefore, a suitable combined MCDM approach should be applied to solve such a complicated problem. In this article, we propose a new method using Fuzzy and credibility theory to allocate the appropriate weights of the criteria, Graph theory and $\mathrm{ABC}$ analysis to determine the best alternative. This method was applied to a real problem and its stages were clarified step by step. The proposed methodology of this study is easy to implement and quite reliable for ranking the alternatives. The findings provide valuable insights for logistics practitioners, academicians, and educators. Future studies could focus on using other decision making methods such as ELECTERE, and VICOR. Based on the context of the problem, and for evaluating more realistic problems, Triangular and Trapezoidal fuzzy numbers could be used. 
And also other methods such as BORDA or the liner allocation algorithm could be applied for filtering effective attributes in big and complicated problems.

\section{ACKNOWLEDGEMENTS}

The authors would like to thanks for all supported by research deputy of Islamic Azad university-South Tehran branch related this research.and wewould like toexpress our gratitude to the Chair \& Board of ICSMO 2016 conference,especially thechnical members and all colleagues, for their valuable discussions and accessibility.

\section{REFERENCES}

[1] R. Bhatnagar, A. S. Sohal, and R. Millen, "Third party logistics services: A Singapore perspective," International Journal of Physical Distribution and Logistics Management, vol. 29, no. 9, pp. 569 - 587, 1999.

[2] H. T. Liu and W. K. Wang, "An integrated fuzzy approach for provider evaluation and selection in third-party logistics," Expert Systems with Applications, vol. 36, pp. 4387-4398, 2009.

[3] C. H. Cheng and Y. Lin, "Evaluating the best main battle tank using fuzzy decision theory with linguistic criteria evaluation," European Journal of Operational Research, vol. 142, no. 1, pp. 174-186, 2002.

[4] C. C. Lin and Y. T. Liu, "Genetic algorithms for portfolioselection problem with minimum Transaction lots," European Journal of Operational Research, vol. 185, no. 1, pp. 393-404, 2008

[5] O. Perron, "Grundlagen fur eine theorie des jacobischen kettenbruchalgorithmus," Math. Ann., vol. 64, 1907, pp. 11-76.

[6] G. Frobenius, Uber Matrizen Aus Nicht Negativen Elementen, Sitzungsberichte Preussische Akademie der Wissenschaft, Berlin, 1912, pp. $456-477$

[7] F. Chatelin, Eigenvalues of Matrices, John Wiley and Sons, Chichester, 1993.

[8] F. R. Gantmacher, Theory of Matrix, New York, Chelsea, 1960.
[9] M. A. Cohen and R. Ernst, "Multi-item classification and generic inventory stock control policies," Production and Inventory Management Journal, vol. 29, no. 3, pp. 6-8, 1988

[10] P. Hautaniemi and T. Pirttil, "The choice of replenishment policies in an MRP environment," International Journal of Production Economics, vol. 59, pp. 85-92,1999.

[11] R. Ramanathan, "ABC inventory classification with multiple-criteria using weighted linear optimization," Computers and Operations Research, vol. 33, pp. 695-700, 2006.

[12] A. Kaufmann and M. M. Gupta, "Introduction to fuzzy arithmetic: Theory and applications," New York, Van Nostrand Reinhold, 1985.

[13] P.A. Raj and D. N. Kumar, "Ranking alternatives with fuzzy weights using maximizing set and minimizing set," Fuzzy Sets and Systems, vol. 105 , pp. $365-375,1999$.

[14] L. Zadeh, "Fuzzy sets a basis for a theory of possibility," Fuzzy Set and System, vol. 1, 1978.

[15] W. J. Jiang, Z. C. Fa, J. Y. Yin, and Z. G. Zhong, "Using the fuzzy multi-criteria model to select the optimal cool storage system for air conditioning," Energy and Buildings, vol. 40, pp. 2059-2066, 2008.

[16] L. Zadeh, "A theory of approximate reasoning," Mathematical Frontiers of the Social and Policy Science, Westview Press, Boulder, Colorado, 1979, pp. 69-129.

[17] B. Liu and Y. Liu, "Expected value of fuzzy variable and fuzzy expected value models," IEEE Transactions on Fuzzy Systems, vol. 10 , no. 4 , pp. 445-450, 2002.

[18] J. J. Buckley, "Fuzzy hierarchical analysis," Fuzzy Sets and Systems, vol. 17 , no. 3 , pp. $233-247,1985$

Amir Abbas Shojaie was born in 1970, Tehran, Iran. He has received his $\mathrm{PhD}$ in industrial engineering industrial eng. from Tehran Research and Science University. He is an assistant professor and he has published many academic articles. His current research interests are in project management and project planning, facility layout and location and quality management and quality control etc. 